\title{
Otitis bovina por Rhabditis bovis en Córdoba, Colombia. Reporte de dos casos
}

\author{
Bovine otitis by Rhabditis bovis in Cordoba, colombia. \\ Report of two cases
}

\author{
José Cardona Á, ${ }^{* 1}$ M.Sc, Marco González T, ${ }^{1}$ M.Sc, Jaime Álvarez P, ${ }^{1}$ M.Sc. \\ ${ }^{1}$ Universidad de Córdoba, Facultad de Medicina Veterinaria y Zootecnia, Departamento de Ciencias \\ Pecuarias. Montería, Colombia.*Correspondencia: cardonalvarez@hotmail.com
}

Recibido: Enero 5 de 2010; Aceptado: Noviembre 24 de 2010.

\section{RESUMEN}

Se reportan dos casos de bovinos de la raza Gyr puros, atendidos en el servicio ambulatorio de la clínica medico-quirúrgica de grandes animales de la Facultad de Medicina Veterinaria y Zootecnia de la Universidad de Córdoba, Colombia. Los animales presentaron al examen físico signos de otitis clínica en ambos lados, con otorrea, olor fétido, inflamación con cierto grado de estenosis del conducto auditivo, inclinación de la cabeza, prurito intenso y rascado excesivo de las orejas. Se realizó inspección directa y colecta de material del conducto auditivo externo, y se observó en forma directa el movimiento de los parásitos en el cerumen, posteriormente confirmado por observación microscopio-estereoscópico, en el laboratorio de parasitología de la Facultad de Medicina Veterinaria de la Universidad de Córdoba, en el cual se reveló la presencia de larvas y adultos de nemátodos del género Rhabditis bovis. Es el primer reporte de esta enfermedad en bovinos de la raza Gyr en el departamento de Córdoba y Colombia.

Palabras clave: Otitis parasitaria, Rhabditis bovis, bovinos.

\section{ABSTRACT}

Two cases of pure Gyr bred bovines, treated at the outpatient medical-surgical clinic for large animals of the Faculty of Veterinary Medicine and Animal Husbandry, at the University of Cordoba, Colombia are reported. During the physical examination, the animals showed signs of clinical otitis with otorrhea, fetid odor, swelling with some degree of stenosis of the ear canal, head inclination, intense itching and excessive scratching of both ears. Direct inspection was performed and a collection of material from the outer ear canal was taken, the 
movement of parasites in the wax was directly observed, and later confirmed by observation under a stereoscopic microscope in the Parasitology laboratory of the Faculty of Veterinary Medicine at the University of Cordoba, which revealed the presence of nematode larvae and adults of the genus Rhabditis bovis. This is the first report of this disease in Gyr cattle in the department of Cordoba and in Colombia.

Key words: Parasitic otitis, Rhabditis bovis, bovine.

\section{INTRODUCCIÓN}

Las otitis parasitaria bovina causada por nematodos Rhabditiformes ha sido reportada en diversos países africanos de clima caliente y húmedo como Tanzania (1), Kenia $(2,3)$ y Zimbabwe $(4)$, sin embargo la mayoría de los reportes de esta enfermedad se han generado en Brasil (5-9).

Las manifestaciones clínicas de un bovino con otitis parasitaria incluyen otitis clínica en el conducto auditivo, otorrea que puede llegar a ser purulenta, olor fétido, inflamación con cierto grado de estenosis del conducto auditivo, prurito intenso con rascado excesivo de las orejas. Algunos animales se pueden observar con la cabeza pendulante de un lado a otro, por el constante balanceo de las orejas $(8,9)$, en ocasiones ciertas otitis pueden llegar a producir síndrome vestibular y en algunos casos puede ocurrir lesión del nervio facial $(10,11)$. Por otro lado a la inspección del conducto auditivo externo y colecta del cerumen, se puede observar en forma directa el movimiento de los parásitos (8).

La raza Gyr parece estar predispuesta a la otitis parasitaria (Rhabditis bovis) en relación con otras razas, debido a la conformación anatómica del pabellón auricular, el cual es bastante largo y encartuchado, lo que favorece la retención de cerumen proporcionando un ambiente favorable para la reproducción y permanencia del parasito, condición favorecida por la presencia de pelos y proliferación de bacterias saprofitas (12-14); Sin embargo Leite et al (12) y Vieira et al (13), reportaron la otitis parasitara en la raza Indubrasil.

Son considerables las pérdidas económicas en las explotaciones de zonas tropicales y subtropicales, en lo referente a los gastos de medicamentos, manejo, disminución en la producción y ganancia de peso $(9,14)$.

Con respecto a la epizootiología del parasito, se reporta que una de la fuentes de contagio son las actividades grupales como baños contra ectoparasitos y sitios de libre desplazamiento y contacto de animales enfermos con otros animales sanos $(15,16)$. Por otro lado, se reporta que uno de los factores de riesgo relevantes es la presencia exagerada de moscas, así como las épocas lluviosas que también favorecen la presentación de la enfermedad (4).

En el presente reporte, se describen hallazgos clínicos y diagnósticos de dos casos de otitis parasitaria bovina por Rhabditis bovis en dos bovinos de ambos sexos de la raza Gyr en el departamento de Córdoba, Colombia.

\section{CASO CLÍNICO}

Fueron atendidos en el servicio ambulatorio de la clínica medico-quirúrgica de grandes animales de la Facultad de Medicina Veterinaria y Zootecnia de la Universidad de Córdoba, Colombia, dos bovinos de la raza Gyr, un macho de 32 meses de edad, con un peso de $400 \mathrm{~kg}$ y una hembra de 28 meses de edad y un peso de 280 $\mathrm{Kg}$, los cuales presentaron otorrea con secreción purulenta (Figura 1), con olor fétido, inflamación y estenosis de los conductos auditivos (Figura 2), prurito intenso, balanceo constante de la cabeza y rascado excesivo de las orejas, así como linfadenitis a nivel de los nódulos linfáticos mandibulares, los demás parámetros fisiológicos se encontraban dentro de los valores normales (temperatura, tiempo 
de Ilenado capilar, frecuencia ruminal, cardiaca y respiratoria). Según lo reportado por los propietarios en la anamnesis, los animales tenían aproximadamente 8 meses de presentar algunas manifestaciones clínicas como: pérdida progresiva de peso, mal olor y rascado constante en las orejas, también informaron que había

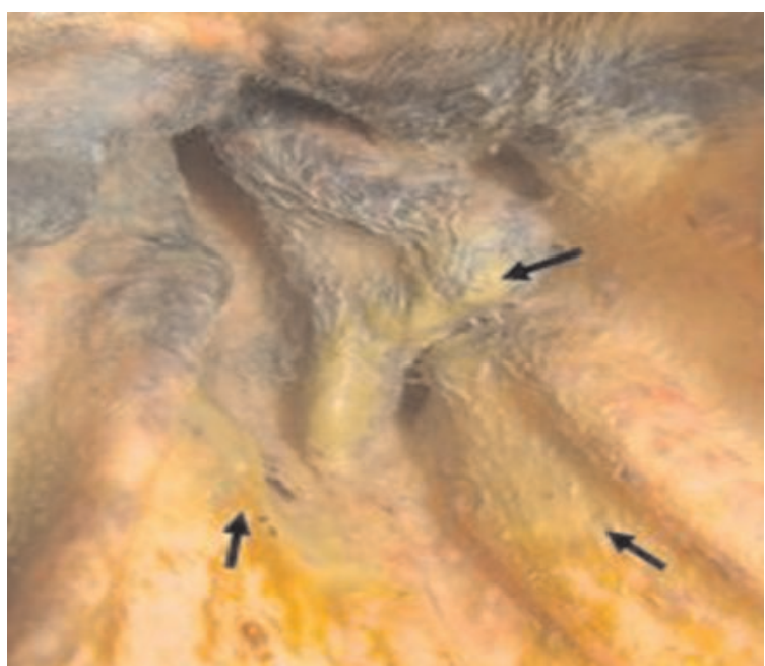

Figura 1. Otorrea (exudado), acompañada de material purulento $(\circledR)$.

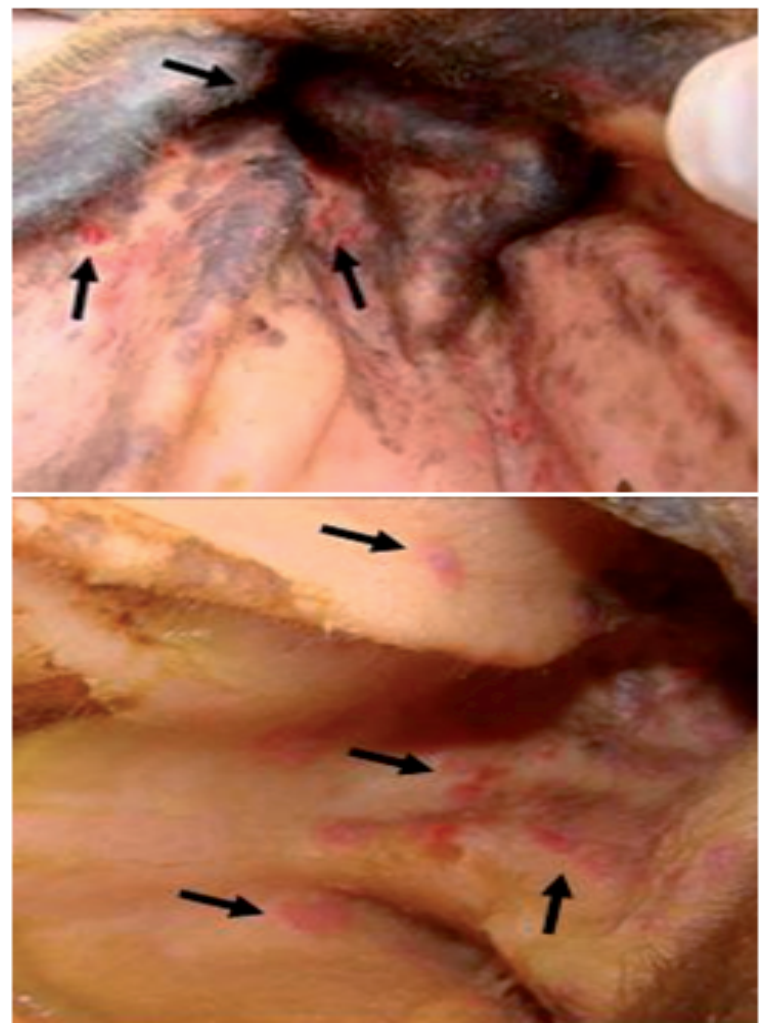

Figura 2. Apariencia del conducto auditivo externo con lesiones inflamatorias y estenosis del conducto auditivo externo $(\circledR)$. sido diagnosticado y tratado como micosis ótica, sin mostrar mejoría al tratamiento antimicótico instaurado.

Los hallazgos clínicos encontrados sugirieron un cuadro de otitis clínica, por lo que se le realizó colecta de material del conducto auditivo externo, con una sonda acanalada (Figura 3) y se observó en forma directa el movimiento de los parásitos en el cerumen, posteriormente fue observado en el laboratorio de parasitología de la Facultad de Medicina Veterinaria de la Universidad de Córdoba, por análisis estereoscópico y microscópico, en el cual se reveló la presencia de larvas y adultos de nemátodos del género Rhabditis bovis (Figura 4) agente reportado en la literatura como el causante de la otitis parasitaria bovina (17).
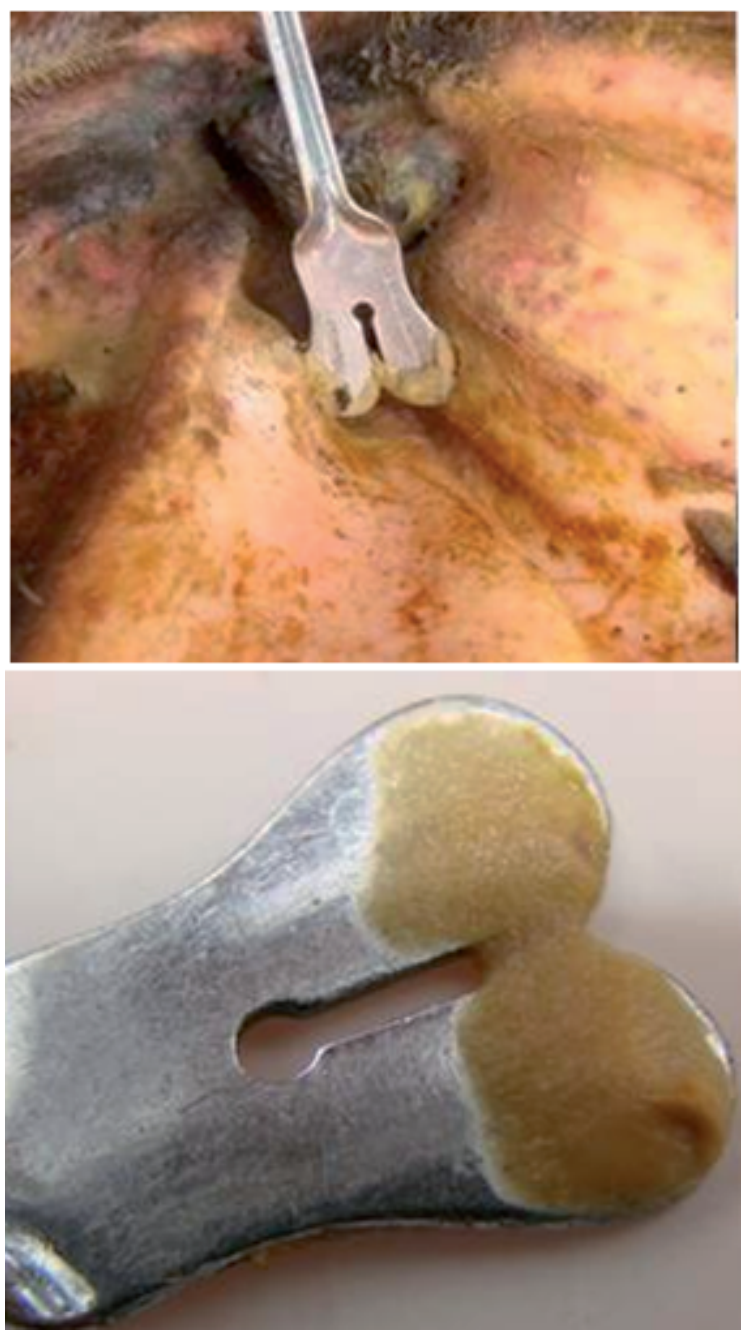

Figura 3. Colecta de cerumen desde el oído externo con sonda acanalada. 


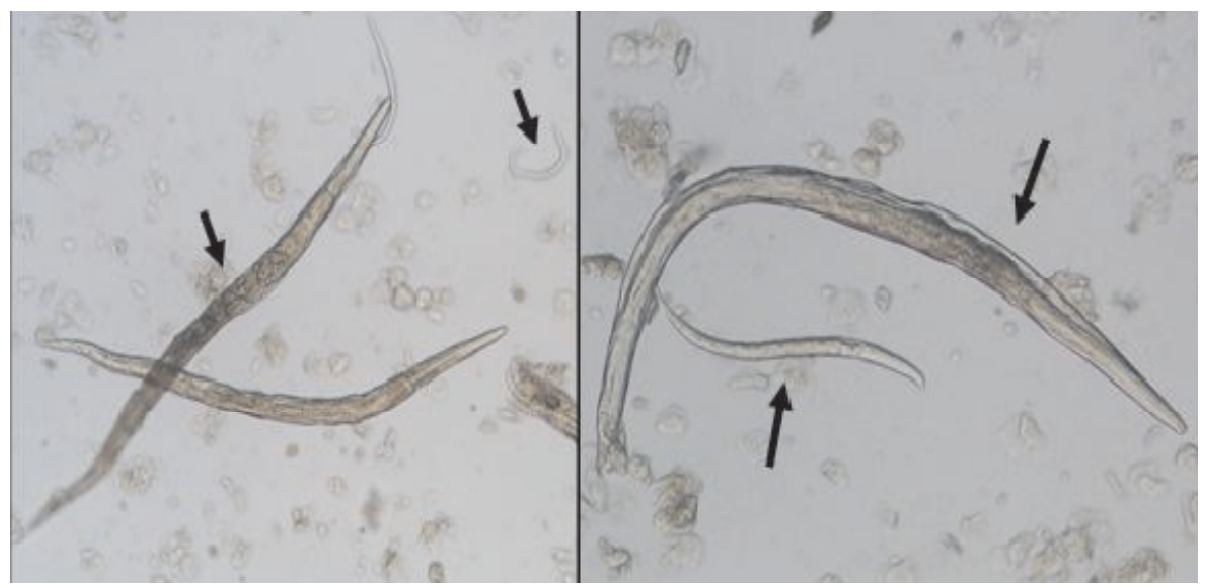

Figura 4. Rhabditis bovis $(\circledR)$, observado en el microscopio. 4x.

\section{DISCUSIÓN}

Los hallazgos clínicos (otorrea, olor fétido, otitis clínica) encontrados en los 2 animales, la anamnesis, el tiempo de padecer la enfermedad y la evolución del cuadro, así como movilidad masal del cerumen son datos importante que hacen presumir o sospechar clínicamente de una otitis parasitaria bovina (Rhabditis bovis) $(14,17)$.

Santos et al (17), indicaron que esta condición parasitaria es común en animales de la raza Gyr e Indubrasil, principalmente en rebaños que se encuentran en países de bosque húmedo tropical. Vieira et al $(13,14)$, describen que los animales con otitis parasitaria en la mayoría de los casos presentan la afección en ambas orejas, y acompañados principalmente de manifestaciones clínicas como depresión, inapetencia, otorrea con secreción algunas veces purulenta y fétida, dolor a la palpación en las bases de los orejas, linfadenitis de los nódulos linfáticos mandibulares.

Algunos autores como Leite et al (6), Duarte et al (7), Vieira et al (14) y Santos et al (17), recomiendan que se debe colectar material de secreción de los conductos auditivos externos y observar el movimiento en olas del material, generado por el movimiento de los parásitos. También indican que se debe transportar el material diluido en solución salina fisiológica a temperatura ambiente para observarlo al microscopio y determinar la presencia de formas parasitarias en estado adulto y larvario de Rhabditis bovis, siendo esta observación la confirmación de la presunción clínica de otitis parasitaria bovina, de igual forma estas recomendaciones se hicieron en los dos casos clínicos informados, para poder reportar la presencia de otitis parasitaria bovina en el departamento de Córdoba, Colombia.

El género Rhabditis, es un parasito saprofito que habita en zonas de bosque húmedo tropical, vive comúnmente en materias fecales, tierras húmedas y materia orgánica en descomposición $(8,14)$, por otro lado Abdala et al (10) y Souza et al (11) reportan que por las características propicias de la oreja de los bovinos de la raza Gyr, se pueden presentar con frecuencia, recidivas de la enfermedad.

En conclusión, por las manifestaciones clínicas (antecedentes, rascado, inquietud, otorrea), las características del cerumen del conducto auditivo externo (color, olor y movimiento), así como la observación del parasito en el laboratorio, permitió diagnosticar otitis parasitaria bovina (Rhabditis bovis) en bovinos de la raza Gyr, en el departamento de Córdoba, Colombia. 


\section{REFERENCIAS}

1. Msolla W, Semuguruka A, Kasuku A, Shoo M. Clinical observations on bovine parasitic otitis in Tanzania. Trop Anim HIth Prod 1993; 25:15-18.

2. Round $M$. The helminth parasites of domesticated animals in Kenya. J Helminthol 1962; 36(4):375-449.

3. Matandala M, Mugera G, Ngatia T. Prevalence of bovine (nematodes) otitis in Kenya. The Kenya Veterinarian 2002; 25:32-35.

4. Ushewokunze-Obatolu U, Pfukenyi D, Ushe T. A retrospective epidemiological study of parasitic otitis in cattle in South-East Lowveld of Zimbabwe. Zimbabwe Veterinary Journal 1999; $30(1): 19-24$.

5. Leite $R$, Nunes $V$, Nunes $A$, Costa $A$, Faccini J, Lopes C. Otite parasitária por nematóides Rhabditiformes: aspectos epidemiológicos e clínicos. Rev Bras Med Vet 1993; 15(2):49-51.

6. Leite R, Leite C, Faccini J. Diagnóstico e tratamento da otite parasitária por nematóides Rhabditiformes em bovinos Rev Brás Parasitol Vet 1994; 3(1):69-70.

7. Duarte E, Melo M, Hamdan J. Epidemiological aspects of bovine parasitic otitis caused by Rhabditis spp. and/or Raillietia spp. in the state of Minas Gerais, Brazil. Vet Parasitol 2001; 101(1):45-52.

8. Duarte $\mathrm{E}$, Hamdan J. Otitis in cattle, an aetiological review. J Vet Med B 2004; 51:1-7.

9. Verocai G, Fernandes J, Thaís R, Correia R, Melo R, Alves A, Scott F. Otite parasitária bovina por nematóides Rhabditiformes em vacas gir no estado do rio de janeiro, Brasil. Rev Bras Parasitol Vet 2007; 16(2):105-107.

10. Abdalla $M$, Peixoto $T$, Alves $P$, França $T$, Brito M. Aspectos anatomo-patológicos da otite causada por Rhabditis sp. em bovinos no estado do Rio de janeiro, Brasil. Congresso Brasileiro de Veterinária 2008. [Accedida el 10/06/2009]. URL Disponible en:http:// www.sovergs.com.br/conbravet2008/ anais/cd/resumos/R0743-1.pdf.

11. Souza W, Calderaro T, Matosinho R, Pratellesi neto $B$, Soliva A, Neves M. Otite parasitária causada por nematóides Rhabditiformes. Revista científica eletrônica de Medicina Veterinária 2008; 6(11). [Accedida el 09/06/2009]. URL Disponible en: http://www.revista.inf.br/ veterinaria12/revisao/revisao.htm.

12. Leite R, Nunes V, Nunes I, Costa A, Faccini J, Lopes C. Otite parasitária por nematóides Rhabditiformes: aspectos epidemiológicos e clínicos. Ver Bras Med Vet $1993 ; 15(2): 49-51$.

13. Vieira M, Da Silva L, Borges N, Barros J, Iglesias A, Vieira E. Estudo da prevalência de otites clínicas por Rhabidtis sp. em bovinos da raça Gir no estado de Goiás. Anais Esc Agron e Vet 1998; 28(2):19-29.

14. Vieira M, Silva L, Araújo J, Andrade M, Fioravanti M, Silva E. Otites parasitárias por nematóides Rhabditiformes em bovinos: avaliação de tratamentos. Ciênc Anim Bras 2001; 2(1):51-55.

15. Msolla P, Matafu E, Monrad J. Epidemiology of bovine parasitic otitis in Tanzania. Trop Anim HIth Prod 1986; 18(1):51-52.

16. Msolla P, Mmbuji W, Kasuku A. Field control of bovine parasitic otitis. Trop Anim HIth Prod 1987; 19:179-183.

17. Santos S, Serôdio J, Silva D, Silva T, Prado T, Brianezi V, Franco da Silva L. Evolução clínica, diagnóstico, tratamento e achados de necropsia da otite parasitária por Rhabditis sp. em touro da raça gir - relato de caso. Ciênc Anim Bras 2009; 1:677-683. 